\title{
A Systematic Review of Interventions for Childhood Depression
}

\author{
Yang Chen*
}

\author{
The Hong Kong Polytechnic University \\ Hung Hom, Kowloon, Hong Kong \\ *Corresponding author.Email: chenyang@shenzhen.com./745867412@qq.com
}

\begin{abstract}
The incidence rate of childhood depression has been rising in the past few years, but the information and consciousness about it have not been popular at the same speed. The purpose of this paper is to describe the etiology, diagnosis and major interventions of depression in childhood. Although it is interlinked with adult depression, the externalization performance is more obvious, the diagnosis is relatively complex, and the interventions or treatments are also interesting and gentle because of immature development of children's mental and physical. Suitable cure methods should be chosen in line with different traits of childhood depression. A literature review was performed using "intervention/ treatment, depression, children/childhood" through Google Scholar for the period time from 2010 to 2021, which can provide a reference for further study of childhood depression, and also be a review of this kind of illness for other scholars.
\end{abstract}

Keywords: Childhood, Depression, Intervention.

\section{INTRODUCTION}

Depression is a common mental health illness that people are familiar with. However, more than 30 years ago, it was considered to occur in adults mainly, children were unlikely to experience depression because of immature development, emotional "loss" was just seen as a part of their "normal" emotional fluctuations. Unfortunately, the morbidity of childhood depression is increasing with the progress of society. The prevalence of depression is estimated to be $2.8 \%$ in children younger than 13 years and $5.6 \%$ in adolescents 13 to 18 years of age. Approximately $60 \%$ of adolescents with depression have recurrences throughout adulthood [1]. As a significant public health issue nowadays, childhood depression deserves much more special attention, considering its acute and lasting consequences associated with depressive disorder such as the severity and suicide risk.

On the basis of the Behavior Assessment System for Children, the symptoms of childhood depression are unhappiness, sadness, and stress, which may lead to the inability to daily activities or even cause suicide. Historical models of etiology for childhood depression largely originated as adaptations of adult models. Recently, a multidimensional, developmental, transactional perspective in and between each of the child's ecologies. Thus, prominent models include biological (e.g., neurochemistry), cognitive (e.g., attributions), behavioral/interpersonal (e.g., interpersonal relationships, social problems), family (e.g., parent-child dyad, attachment theory), and life stress (e.g., stress exposure, diathesis-stress) [2]. All the factors interact dynamically over time to create the onset of childhood depression. Unlike adults, young children typically show signs of depression through their outward appearance and physical complaints. The most universal psychosomatic complaints of children include stomachaches or nausea, headache, pain in the eyes, pain in the limbs or joints, and tingling sensations or numbness. In addition, the failure to make developmentally appropriate weight gain is associated with childhood depression[3].

Diagnosis of childhood depression is complex since the DSM-IV-TR does not offer a classification of childhood depression under the category of "Disorders Usually First Diagnosed in Infancy, Childhood or Adolescence" on the basis of some studies. And the description of diagnostic criteria for depression cannot distinguish between children and adolescents, whose symptoms just only mentions briefly to indicate the difference among children. Sometimes, differential diagnosis requires a long time of symptom examination. For example, Waterman and Ryan (1993) reported that $20-30 \%$ of children diagnosed with depression, when 
followed over time, prove to have bipolar disorder. Therefore, it is a complicated process containing removing incorrect diagnosis and discovering comorbidity, because comorbidity can make an effect on the recurrence of depressive episodes, suicidality, daily functioning, and also the response to treatment and the use of mental health services[4].

An exact diagnosis is significant for a cure plan. A couple of researches showed that a combination of psychotherapy and medication is the most effective treatment of childhood depression. Several studies supported that the most valid psychotherapy for 7 to 14 years old children with depression is cognitive behavioral therapy, psychological education, social skills development, social ability training, self-control therapy, cognitive reconstruction and problem-solving technology, etc[5]. While Some experts have also indicated that a multi-systematic means, including families, mental health practitioners, schools and doctors as the best treatment for childhood depression.

In short, Childhood depression is a serious mental health disease, which increases the risk of long-term and severe depression, and even suicide with untreated time. But a large number of studies are still exploring the causes and treatments of depression in adults yet. Thus, the present study aims to conduct a systematic review of interventions for childhood depression. Due to the diversity and complexity, it only selected four major and common interventions for children who are under 13 years old to review and discuss in this paper.

\section{METHODOLOGY}

All the information are collected from Google scholar, using the terms "intervention/ treatment, depression, children/childhood", with restriction of the date from Jan 2010 to Apr 2021, prescribing a limit to the language of English and appearing in the title of articles, excluding patents and citation. There are 263 records in total.

\section{ELIGIBILITY CRITERIA}

According to the aim of this review paper, the following eligibility criteria would be suitable for screening procedures.

1. The article must be available, peer viewed and fulltext.

2. The article must report original empirical results.

3. The research object of the article must be human participants.

4. The article used interventions or treatments for children with depression.

Finally, 9articles were included in this review.

\section{RESULTS}

Lenze. et al.[6] created an Emotional Development (ED) module, which was integrated with an established invention called Parent-Child Interaction Therapy (PCIT) to solve emotion development problems of preschool depression suffers. This module laid particular emphasis on teaching parents to promote children's emotional development and enhance emotional regulation. There are eight parent-child dyads with depressed preschoolers participating in 14 sessions of treatment. PCIT-ED includes three codes: (a) standard PCIT, which aims at the parent-child relationship, using behavioral and play therapy techniques to improve relationship quality and parents' ability to set parenting and effective restrictions. (b) key components: Child-Directed Interaction (CDI) and Parent Directed Interaction (PDI). The former focuses on strengthening the parent-child relationship by teaching positive game skills, while the later is concentrated on reducing destructive behavior by teaching parents to give effective orders and training parents to deal with non-conforming behaviors. The result showed that PCIT-ED is a promising intervention for preschool depression patients, significantly reducing internalizing and externalizing symptoms as well as functional impairment before and after treatment.

Nash, N[7] evaluated the relationship between Resilience and Coping Intervention (RCI) and resilience among 200 foster children, who are 10 to 16 years old with a medical fragile classification by DCFS and diagnosed depression, as well. The intervention of RCI can help individuals to identify their thoughts, feelings and coping strategies, and also can be good for the resilience in foster children. Participants were divided into 10 intervention groups and 10 control groups, both of whom were required to answer the pre-test (paperpencil) questionnaire one week before the intervention. The control groups contained games, movies, painting, art and craft, for a total of 4 sessions. The intervention groups received RCI once a week for 4 weeks in one hour sessions (a total of 4 sessions), which was conducted by a discussion leader and an assistant trained in RCI management. According to the result, RCI can contribute to the resilience of foster children through measuring by Children's and Adolescent Coping Strategies Checklist (CCSC), Child Strength and Difficulties Questionnaire (CSDQ), and The Hope Questionnaire (HQ).

Patras, J. et al.[8] assessed the changes in depressive symptoms by a randomized controlled trial, whose participants aged 8 to 11 from 36 schools. It was divided into two situations: (a) group cognitive behavior intervention EMOTION or (b) treatment as usual (TAU). The evaluation was conducted at pre-, mid - intervention, post-, and one year after the intervention. The children completed the self-report questionnaire, and the parents and teachers reported them. The measures were the Short Mood and Feelings Questionnaire (SMFQ) and 
Multidimensional Anxiety Scale for Children (MASC). Observers will estimate the quality of implementation through fidelity rating based on the video recording of the team leader who leads the emotional group meeting. The results indicated that the EMOTION program is an effective intervention for the prevention of later depression in children.

Kösters, M. P.et al.[9] investigated the effect of FRIENDS for Life as a school-based intervention in the prevention of childhood depression under a naturalistic environment. 496 children aged 8-13 were differentiated into the intervention group and control group in a quasiexperiment. The depression scores of self-, teachers and peers before and after intervention during some certain time were collected for assessment. FRIENDS for Life program is a protocol-led preventive intervention program for children's depression based on cognitive behavioral therapy, which teaches children how to recognize symptoms of depression, how to relax, how to act when problems arise, how to engage in positive selfdialogue, how to gradually expose themselves to fear, and how to reward themselves after trying instead of focusing on success. The results indicated that FRIENDS for Life is a targeted prevention plan, which can reduce childhood depression problems for a continuous-time after the implementation in daily school practice.

Furthermore, there is also a couple of review literature mentioned that the intervention of childhood depression includes traditional drug therapy, play therapy, family cognitive behavior therapy, parent-child interaction therapy and all the rest of it. It is found that despite the treatment of depression in young people is currently controversial, medication remains an important part of the treatment approach for this disorder, although the risks and benefits of pharmacotherapy need to be carefully assessed in each patient[10]. Parent-child interaction therapy and play therapy play a vital important role in improving the parent-child relationship and the efficacy of depression, either. Each literature has made a detailed description of the effectiveness of the intervention mentioned above, and it will not be repeated in the present paper.

Table 1 Summary of Perspectives on Intervention of Children

\begin{tabular}{cccc}
\hline Authors (Year) & N & Interventions & Main Results \\
\hline Lvenze et al (2011) & 8 families & PCIT-ED & Internalizing and externalizing \\
Nash, N. (2020) & 200 samples & RCl & Rymptoms decreased \\
Patras, J. et al (2016) & 36 schools & Emotion Program & Reducing symptoms of depression \\
Kösters, M. P.et al (2015) & 496 children & FRIENDS for Life & yielding long-lasting and continuing \\
& & & reduction in depression problems
\end{tabular}

Note: N: The Number of Participants; PCIT-ED: Parent-child Intervention Therapy Emotional Development; RCI: Resilience and Coping Intervention.

\section{DISCUSSION}

This current review aims to summarize the intervention and effects of childhood depression. In order to arouse public attention to childhood depression, it focused on four frequent interventions in this passage, which is going to provide a reference for the treatment of childhood depression in the future.

In line with the literature review, PCIT-ED, which combined with parent-child interaction therapy, can strengthen the parent-child relationship by teaching parents behavior and games technology, and reduce the internalization and externalization symptoms and functional damage of childhood depression. RCI can improve psychological resilience and promote the recovery of foster children with depression by assisting them in identifying their thoughts, emotions and coping strategies. Emotion Program can ease the symptoms of depression through group cognitive-behavioural emotional intervention, which is appraised by Short Mood and Feelings Questionnaire (SMFQ) and Multidimensional Anxiety Scale for Children (MASC). Friends for life is a cognitive therapy-based intervention, which instructs suffers how to understand childhood depression correctly and treat it lightly in a proper way, mitigating the symptoms of childhood depression for a long-term, continuous period. 
In summary, the treatment of childhood depression should be based on individual clinical symptoms and the actual situation.

Like any other study, this study is not free from limitations. At present, there is still a lack of research on a certain treatment for childhood depression, and the literature is still unavailable so that a comprehensive review cannot be made. In the future, more and deep research of the treatment in childhood depression need to apply and test.

\section{CONCLUSION}

Depression has become the second-largest disease in the world, and it is gradually endangering children's physical and mental health nowadays, which must cause widespread concern and high attention the society. The interventions vary since the etiology and diagnosis between childhood depression and adult depression are distinguished. And because children are not mature yet, it will achieve the best curative effect to adopt interesting and simple intervention which more suitable for children's psychological and physiological development characteristics, supplemented with drug treatment as appropriate.

\section{REFERENCES}

[1] Clark, M. S., Jansen, K. L., \& Cloy, J. A. (2012). Treatment of childhood and adolescent depression. American family physician, 86(5), 442448.

[2] Ruderman, M. A., Stifel, S. W., O’Malley, M., \& Jimerson, S. R. (2013). The school psychologist's primer on childhood depression: a review of research regarding epidemiology, etiology, assessment, and treatment. Contemporary School Psychology: Formerly" The California School Psychologist", 17(1), 35-49.
[3] Peratikos-Kiritsis, A. (2010). Cognitive Behavioral Play Therapy for children with depression: A manual for individual treatment. University of Hartford.

[4] Abrahamian, A. (2012). Diagnosis and treatment of childhood depression: A resource for pediatricians (Doctoral dissertation, Pepperdine University).

[5] Huberty, T. J. (2012). Anxiety and depression in children and adolescents: Assessment, intervention, and prevention. Springer Science \& Business Media.

[6] Lenze, S. N., Pautsch, J., \& Luby, J. (2011). Parentchild interaction therapy emotion development: A novel treatment for depression in preschool children. Depression and Anxiety, 28(2), 153-159.

[7] Nash, N. (2020). The Effect of Resilience and Coping Intervention (RCI) on Resilience among Medical Fragile Foster Children with Depression.

[8] Patras, J., Martinsen, K. D., Holen, S., Sund, A. M., Adolfsen, F., Rasmussen, L. M. P., \& Neumer, S. P. (2016). Study protocol of an RCT of EMOTION: An indicated intervention for children with symptoms of anxiety and depression. BMC psychology, 4(1), 1-8.

[9] Kösters, M. P., Chinapaw, M. J., Zwaanswijk, M., Van Der Wal, M. F., \& Koot, H. M. (2015). Indicated prevention of childhood anxiety and depression: Results from a practice-based study up to 12 months after the intervention. American journal of public health, 105(10), 2005-2013.

[10] Dubicka, B., Wilkinson, P., Kelvin, R. G., \& Goodyer, I. M. (2010). Pharmacological treatment of depression and bipolar disorder in children and adolescents. Advances in psychiatric treatment, 16(6), 402-412. 